DESY-96-260

hep-ph/9612415

\title{
Production of Charm Quark Jets in DIS Diffractive Dissociation
}

\author{
H. Lotter \\ II. Institut f. Theoretische Physik, Universität Hamburg, \\ Luruper Chaussee 149, D-22761 Hamburg 1
}

\begin{abstract}
We present a calculation of open charm quark production in diffractive deep inelastic electronproton scattering in a perturbative QCD framework. The cross section is proportional to the square of the gluon density and explicitly displays breaking of Regge factorization. Jet cross sections as well as the charm contribution to the diffractive structure function are calculated. As a consequence of the steep rise of the gluon density at small $x$ the charm contribution to $F_{2}^{D}$ rises with decreasing $x_{\mathbb{P}}$.
\end{abstract}

\footnotetext{
${ }^{1}$ Supported by Bundesministerium für Forschung und Technologie, Bonn, Germany under Contract 05 6HH93P(5) and EEC Program "Human Capital and Mobility" through Network "Physics at High Energy Colliders" under Contract CHRX-CT930357 (DG12 COMA).
} 
1.) In the context of the discussion of rapidity gap events in deep inelastic electron-proton scattering observed at HERA a subclass of diffractive events in which a large mass scale appears in the diffractively produced hadronic final state has created particular interest. Representatives of this type of events are the diffractive production of heavy vector mesons [1], diffractive jet production [2, 3, 4, 5, 6] and diffractive production of open charm [7, 8]. Due to the presence of the large mass scale these processes offer the possibility to apply and test perturbative QCD in the setting of diffractive scattering. The common feature of the above cited processes is the dependence of the cross section on the square of the gluon density of the proton. Because of this strong sensitivity these events have been considered as a possible probe of the gluon density.

In the above list the process of diffractive open charm production is particularly promising. Compared to heavy vector meson production it does not depend on a meson wave function which is poorly determined from the theoretical side. Compared to jet production charm production does not require a large transverse momentum which in turn leads to a strongly suppressed event rate.

In this letter we generalize our preceding calculations on jet production [2, 3] in DIS diffractive dissociation to finite quark mass. This allows, in particular, the investigation of open charm production. Our calculation is based on an analytical expression for the unintegrated gluon structure function which enables us to take into account a subset of subleading corrections which turn out to be numerically important [2]. As the new contribution of the present work we calculate jet cross sections for charm quarks and compare with massless flavours. In addition we compare the magnitude of the jet cross section calculated in our model with the predictions of a model which is based on nonperturbative two-gluon exchange 《. for the elecron-proton cross section include the dependence of the cross section on the angle of the jet plane relative to the electron plane. For the case of the large charm mass we can extend our expressions to low transverse momenta and can even integrate the transverse momentum to obtain the charm contribution of the diffractive structure function. We discuss the $x_{\mathbb{P}}, \beta$ and $Q^{2}$ dependence of the charm contribution to $F_{2}^{D(3)}$. In this part of our analysis we obtain results similar to the ones presented in [7] and [8].

2.) The kinematics of the process is well-known and we only give the key ingredients. To calculate first the hadronic tensor $H_{\mu \nu}$ consider the photon-proton subprocess $\gamma^{*}(q)+P(p) \rightarrow c(k)+\bar{c}(q-k+\Delta)+P(p-\Delta)$. We define the momenta of the particles as indicated in figure 11. The calculation is performed for zero

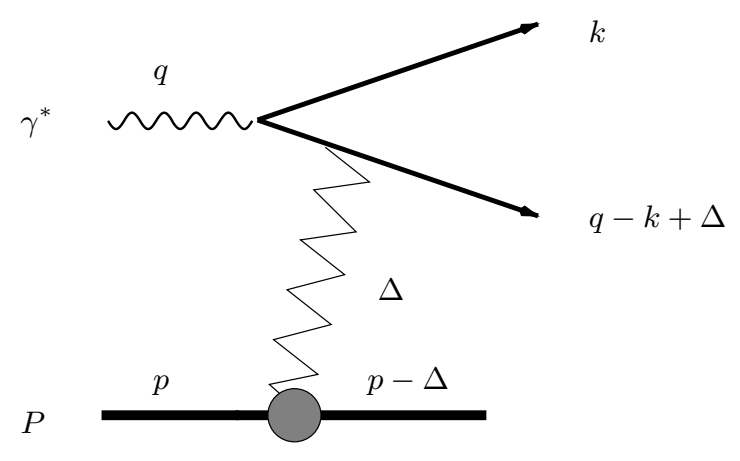

Figure 1: Diffractive production of a quark-antiquark pair in photon-proton scattering.

momentum transfer $t=\Delta^{2}=0$. For the momenta $k$ and $\Delta$ we use a Sudakov decomposition w. r. t. the light cone momenta $p$ and $q^{\prime}=q+x p\left(x=Q^{2} /(2 p q)\right)$

$$
\begin{aligned}
k & =\alpha q^{\prime}+\beta p+\mathbf{k}, \\
\Delta & =\alpha_{\Delta} q^{\prime}+x_{\mathbb{P}} p+\Delta .
\end{aligned}
$$

Using the mass shell conditions for the outgoing particles one can show that we have $\alpha_{\Delta}=\mathcal{O}\left(\boldsymbol{\Delta}^{2} /\left(2 p q^{\prime}\right)\right)$. Assuming $\boldsymbol{\Delta}^{2} \ll p q^{\prime}$ and hence neglecting $\alpha_{\Delta}$ and $\boldsymbol{\Delta}$ we can cast the phase space in the form

$$
d \Gamma=\frac{\pi}{8 p q} \frac{1}{\sqrt{1-4 \frac{\mathbf{k}^{2}+m_{c}^{2}}{M^{2}}}} d M^{2} d t d \mathbf{k}^{2}
$$


with the charm quark mass $m_{c}^{2}$ and $M^{2}$ being the invariant mass of the $c \bar{c}$ pair which is related to the light cone momentum fraction $\alpha$ through the relation

$$
M^{2}=\frac{\mathbf{k}^{2}+m_{c}^{2}}{\alpha(1-\alpha)}
$$

Energy-momentum conservation then leads to the phase space restriction $M^{2} \geq 4\left(\mathbf{k}^{2}+m_{c}^{2}\right)$. Furthermore the longitudinal momentum fraction $x_{\mathbb{P}}$ transferred from the proton to the $c \bar{c}$ pair is fixed as

$$
x_{\mathbb{P}}=\frac{M^{2}+Q^{2}}{W^{2}+Q^{2}} .
$$

$W^{2}=(p+q)^{2}$ is the cms-energy of the photon-proton system. Another often used variable is $\beta$ defined as

$$
\beta=\frac{Q^{2}}{Q^{2}+M^{2}}
$$

from which follows $\beta=x / x_{\mathbb{P}}$.

Now we have to specify the coupling of the $c \bar{c}$ pair to the proton. As the simplest model for an interaction in which no color is transferred from the proton to the $c \bar{c}$ pair we take two-gluon exchange. Since the charm quark is sufficiently heavy we treat both gluons perturbatively. We then make use of high-energy factorization [9] to express the amplitude of the photon-proton subprocess in terms of the unintegrated gluon density of the proton (fig. 2)

$$
H_{\mu \nu}=\left(\int d^{2} \mathbf{l} C_{\mu}\left(\mathbf{l} ; \mathbf{k}, Q^{2}, M^{2}\right) \mathcal{F}_{G}\left(x_{\mathbb{P}}, \mathbf{l}^{2}\right)\right)\left(\int d^{2} \mathbf{l}^{\prime} C_{\nu}\left(\mathbf{l}^{\prime} ; \mathbf{k}, Q^{2}, M^{2}\right) \mathcal{F}_{G}\left(x_{\mathbb{P}}, \mathbf{l}^{\prime 2}\right)\right)^{*} .
$$

This factorization is valid in the leading- $\log \left(1 / x_{\mathbb{P}}\right)$ approximation in which the imaginary part of the diagrams in fig. 2 contributes. In this approximation the difference of the longitudinal momenta of the two gluons is neglected. It is therefore legitimate to use the same diagonal gluon density $\mathcal{F}_{G}\left(x_{\mathbb{P}}, \mathbf{l}^{2}\right)$ which appears in inclusive DIS. The coefficient function $C_{\mu}$ represents the upper part of the diagrams in fig. 2 with

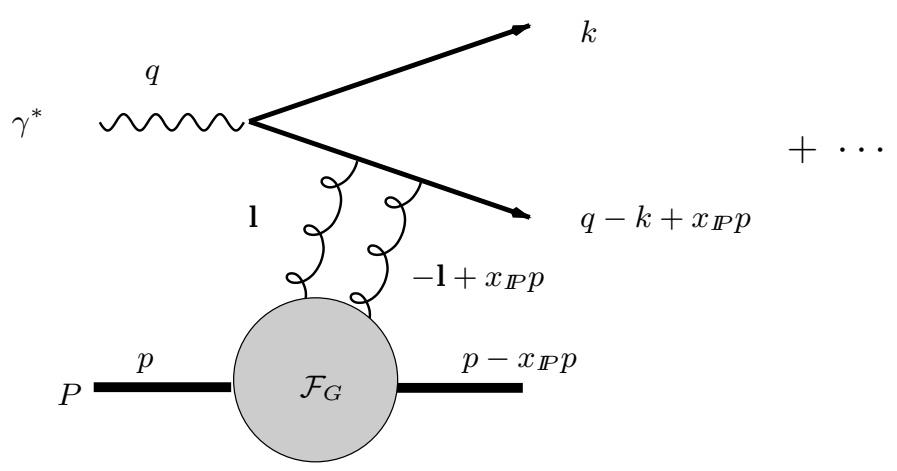

Figure 2: Representation of the amplitude in terms of the unintegrated gluon structure function. The dots represent three other diagrams which are generated by attaching the gluons to the quark lines in all possible ways.

the gluons coupled to the quarks in all possible ways. The calculation of the respective diagrams is straightforward if one uses the fact that the gluon polarization proportional to $p^{\sigma}$ gives the dominant contribution in the small- $x$ limit 10, 11, 12.

In order to obtain the electron-proton cross section we have to contract $H_{\mu \nu}$ with the usual lepton tensor. 
This yields the following expression

$$
\begin{aligned}
\frac{d \sigma^{e P}}{d y d Q^{2} d M^{2} d \mathbf{k}^{2} d \phi d t}{ }_{\mid t=0}=\frac{\alpha_{\mathrm{em}}}{2 y Q^{2} \pi^{2}} & {\left[\left.\frac{1+(1-y)^{2}}{2} \frac{d \sigma_{T_{1}}^{\gamma^{*} P}}{d M^{2} d \mathbf{k}^{2} d t}\right|_{t=0}-\left.2(1-y) \cos 2 \phi \frac{d \sigma_{T_{2}}^{\gamma^{*} P}}{d M^{2} d \mathbf{k}^{2} d t}\right|_{t=0}\right.} \\
& \left.+\left.(1-y) \frac{d \sigma_{L}^{\gamma^{*} P}}{d M^{2} d \mathbf{k}^{2} d t}\right|_{t=0}+\left.(2-y) \sqrt{1-y} \cos \phi \frac{d \sigma_{I}^{\gamma^{*} P}}{d M^{2} d \mathbf{k}^{2} d t}\right|_{t=0}\right] .
\end{aligned}
$$

The indices $T$ and $L$ refer to the contributions of transversely and longitudinally polarized photons. The term with index $I$ is the interference contribution. The angle $\phi$ is defined in the two-dimensional transverse plane through the scalar product $\mathbf{e} \cdot \mathbf{k}_{1}=|\mathbf{e}||\mathbf{k}| \cos \phi$. Here $\mathbf{e}$ is the transverse component of the electron momentum $e_{\mu}=1 / y q_{\mu}^{\prime}+x(1-y) / y p_{\mu}+\mathbf{e}_{\mu}$ and $\mathbf{k}_{1}$ is the transverse momentum of the charm (anti)quark which points into the proton hemisphere (in the $c \bar{c}$ cm-system). Note the negative sign of the $\cos 2 \phi$-term which is a characteristic feature of the two-gluon exchange model. In photon-gluon fusion (one-gluon exchange) this term comes with positive sign [3].

The expressions for the subprocess cross sections read

$$
\begin{aligned}
\left.\frac{d \sigma_{T_{1}}^{\gamma^{*} P}}{d M^{2} d \mathbf{k}^{2} d t}\right|_{t=0}= & \frac{1}{M^{4}} \frac{1}{\mathbf{k}^{2}} \frac{1}{12} e_{c}^{2} \alpha_{\mathrm{em}} \pi^{2} \alpha_{s}^{2} \frac{\mathbf{k}^{2}+m_{c}^{2}}{\sqrt{1-4 \frac{\mathbf{k}^{2}+m_{c}^{2}}{M^{2}}}}\left[\left(1-2 \frac{\mathbf{k}^{2}+m_{c}^{2}}{M^{2}}\right)\left[I_{T}\left(Q^{2}, M^{2}, \mathbf{k}^{2}, m_{c}^{2}\right)\right]^{2}\right. \\
& \left.+m_{c}^{2} \frac{\mathbf{k}^{2} M^{4}}{\left(\mathbf{k}^{2}+m_{c}^{2}\right) Q^{4}}\left[I_{L}\left(Q^{2}, M^{2}, \mathbf{k}^{2}, m_{c}^{2}\right)\right]^{2}\right] \\
\left.\frac{d \sigma_{L}^{\gamma^{*} P}}{d M^{2} d \mathbf{k}^{2} d t}\right|_{t=0}= & \frac{1}{M^{4}} \frac{1}{Q^{2}} \frac{4}{3} e_{c}^{2} \alpha_{\mathrm{em}} \pi^{2} \alpha_{s}^{2} \frac{\mathbf{k}^{2}+m_{c}^{2}}{\sqrt{1-4 \frac{\mathbf{k}^{2}+m_{c}^{2}}{M^{2}}}}\left[I_{L}\left(Q^{2}, M^{2}, \mathbf{k}^{2}, m_{c}^{2}\right)\right]^{2} \\
\left.\frac{d \sigma_{I}^{\gamma^{*} P}}{d M^{2} d \mathbf{k}^{2} d t}\right|_{t=0}= & \frac{1}{M^{4}} \frac{1}{\sqrt{\mathbf{k}^{2} Q^{2}}} \frac{1}{3} e_{c}^{2} \alpha_{\mathrm{em}} \pi^{2} \alpha_{s}^{2}\left(\mathbf{k}^{2}+m_{c}^{2}\right) I_{T}\left(Q^{2}, M^{2}, \mathbf{k}^{2}, m_{c}^{2}\right) I_{L}\left(Q^{2}, M^{2}, \mathbf{k}^{2}, m_{c}^{2}\right) \\
\left.\frac{d \sigma_{T_{2}}^{\gamma^{*} P}}{d M^{2} d \mathbf{k}^{2} d t}\right|_{t=0}= & \frac{1}{M^{6}} \frac{1}{\mathbf{k}^{2}} \frac{1}{12} e_{c}^{2} \alpha_{\mathrm{em}} \pi^{2} \alpha_{s}^{2} \frac{\left(\mathbf{k}^{2}+m_{c}^{2}\right)^{2}}{\sqrt{1-4 \frac{\mathbf{k}^{2}+m_{c}^{2}}{M^{2}}}}\left[I_{T}\left(Q^{2}, M^{2}, \mathbf{k}^{2}, m_{c}^{2}\right)\right]^{2}
\end{aligned}
$$

The factor $e_{c}$ denotes the charge of the charm quark. The essential dynamics is contained in the universal functions $I_{L}, I_{T}$ for which we have the expressions

$$
\begin{gathered}
I_{L}\left(Q^{2}, M^{2}, \mathbf{k}^{2}, m_{c}^{2}\right)=-\int \frac{d \mathbf{l}^{2}}{\mathbf{l}^{2}} \mathcal{F}_{G}\left(x_{\mathbb{P}}, \mathbf{l}^{2}\right)\left[\frac{Q^{2}}{M^{2}+Q^{2}}-\frac{\left(\mathbf{k}^{2}+m_{c}^{2}\right) Q^{2}}{M^{2} \sqrt{P_{\mathbf{k}, \mathbf{l}}}}\right] \\
I_{T}\left(Q^{2}, M^{2}, \mathbf{k}^{2}, m_{c}^{2}\right)=-\int \frac{d \mathbf{l}^{2}}{\mathbf{l}^{2}} \mathcal{F}_{G}\left(x_{\mathbb{P}}, \mathbf{l}^{2}\right)\left[\frac{2 M^{2} \mathbf{k}^{2}}{\left(\mathbf{k}^{2}+m_{c}^{2}\right)\left(Q^{2}+M^{2}\right)}-1\right. \\
\left.+\frac{\mathbf{l}^{2}+\frac{\mathbf{k}^{2}}{M^{2}}\left(Q^{2}-M^{2}\right)+m_{c}^{2}\left(1+\frac{Q^{2}}{M^{2}}\right)}{\sqrt{P_{\mathbf{k}, \mathbf{l}}}}\right]
\end{gathered}
$$

with $P_{\mathbf{k}, \mathbf{l}}$ being defined as

$$
P_{\mathbf{k}, \mathbf{l}}=\left(\mathbf{l}^{2}+\frac{\mathbf{k}^{2}}{M^{2}}\left(Q^{2}-M^{2}\right)+\frac{m_{f}^{2}}{M^{2}}\left(Q^{2}+M^{2}\right)\right)^{2}+4 \mathbf{k}^{2}\left(\frac{\mathbf{k}^{2}}{M^{2}} Q^{2}+\frac{m_{f}^{2}}{M^{2}}\left(Q^{2}+M^{2}\right)\right)
$$

These expressions simplify considerably in the case of massless flavors $\left(m_{f}^{2}=0\right)$ [2, 3]. To proceed one has to use an explicit model for the unintegrated gluon structure function $\mathcal{F}_{G}$. Within the leading- $\log \left(1 / x_{\mathbb{P}}\right)$ approximation it is consistent to express $\mathcal{F}_{G}$ as the solution of the BFKL equation [13]. The l-integration in eqs. (13) 14 can then be performed analytically. The main outcome of this calculation is the determination of the relevant scale $\Delta^{2}$ of the process which is found to be

$$
\Delta^{2}=\left(\mathbf{k}^{2}+m_{c}^{2}\right) \frac{1}{1-\beta}
$$


If we assume that this effective scale is large (compared to the inverse size of the proton) we can evaluate the integrals in eqs. (14, 13) in the leading- $\log \left(\Delta^{2}\right)$ approximation. Using the relation

$$
\int^{\Delta^{2}} d \mathbf{l}^{2} \mathcal{F}_{G}\left(x_{\mathbb{P}}, \mathbf{l}^{2}\right)=x_{\mathbb{P}} G\left(x_{\mathbb{P}}, \Delta^{2}\right)
$$

we can express the functions $I_{L}, I_{T}$ within this approximation in terms of the gluon density $x_{\mathbb{P}} G\left(x_{\mathbb{P}}, \Delta^{2}\right)$. The result reads

$$
\begin{aligned}
I_{L} & =\frac{\left(\mathbf{k}^{2}+m_{f}^{2}\right) Q^{2}}{\mathbf{k}^{4} M^{2}} \frac{\xi-1}{(1+\xi)^{3}} x_{\mathbb{P}} G\left(x_{\mathbb{P}}, \frac{\mathbf{k}^{2}+m_{c}^{2}}{1-\beta}\right), \\
I_{T} & =\frac{4}{\mathbf{k}^{2}} \frac{\xi}{(1+\xi)^{3}} x_{\mathbb{P}} G\left(x_{\mathbb{P}}, \frac{\mathbf{k}^{2}+m_{c}^{2}}{1-\beta}\right) .
\end{aligned}
$$

Here we have introduced, for brevity, the scaling variable $\xi=\left(\beta+m_{c}^{2} / \mathbf{k}^{2}\right) /(1-\beta)$.

Inserting these expressions into the cross section formulae we obtain the cross section for diffractive $c \bar{c}$ production in DIS in the double leading logarithmic approximation (DLA). In [2] a certain class of corrections to the DLA was obtained in the massless case which turned out to be numerically important in the small- $\beta$ region. The same type of correction can be calculated in the massive case and the result for the correction terms reads

$$
\begin{aligned}
I_{L}^{(c)} & =\frac{\left(\mathbf{k}^{2}+m_{f}^{2}\right) Q^{2}}{\mathbf{k}^{2} M^{2}} \frac{1}{(1+\xi)^{3}}\left(2+(1-\xi) \log \frac{\xi}{1+\xi}\right) \frac{\partial}{\partial \mathbf{k}^{2}} x_{\mathbb{P}} G\left(x_{\mathbb{P}}, \mathbf{k}^{2}(1+\xi)\right) \\
I_{T}^{(c)} & =2 \frac{1}{(1+\xi)^{3}}\left(1-\xi-2 \xi \log \frac{\xi}{1+\xi}\right) \frac{\partial}{\partial \mathbf{k}^{2}} x_{\mathbb{P}} G\left(x_{\mathbb{P}}, \mathbf{k}^{2}(1+\xi)\right)
\end{aligned}
$$

These terms have to be added to the respective contributions $(18)$ and (19). In the next section we turn to the numerical evaluation of our formulae.

3.) As to the numerical evaluation we start with some preliminary remarks. In all subsequent calculations we have integrated over the momentum transfer $t$ after multiplication of the results of 2.) with the elastic proton form factor 14]

$$
F_{P}\left(x_{\mathbb{P}}, t\right)=\frac{4-2.8 \frac{t}{\mathrm{GeV}^{2}}}{4-\frac{t}{\mathrm{GeV}^{2}}}\left(1-\frac{t}{0.7 \mathrm{GeV}^{2}}\right) x_{\mathbb{P}}^{-0.25 \frac{t}{\mathrm{GeV}^{2}}}
$$

For the charm quark mass we have taken the value $m_{c}=1.5 \mathrm{GeV}$ and we have used the one-loop formula for $\alpha_{s}$ evaluated at the scale $\left(\mathbf{k}^{2}+m_{c}^{2}\right) /(1-\beta)$. In a preceding publication [2] we have discussed whether the leading order or the next-to-leading order gluon density should be used for a numerical prediction. We have presented some evidence that the NLO density is appropriate and consequently we perform all calculations with the GRV 15 NLO gluon density.

First we present results for jet cross sections, i. e. we calculate the total electron proton cross section with a lower cutoff $\mathbf{k}_{0}^{2} \geq 2 \mathrm{GeV}^{2}$ imposed on the transverse momentum integration. The other variables are integrated in a region which is adapted to the HERA kinematics, namely we choose the cuts $Q^{2} \geq 10 \mathrm{GeV}^{2}$, $x_{\mathbb{P}} \leq 10^{-2}$ and $50 \mathrm{GeV}<W<220 \mathrm{GeV}$. The results for the integrated cross section are listed in table 1 . For comparison we quote in table 2 also the results which are obtained for three massless quarks [2].

It can be clearly seen that the relative contribution of the charm quarks compared to the massless flavors is dependent on the lower momentum cutoff $\mathbf{k}_{0}^{2}$. The ratio $r=\sigma_{c \bar{c}} / \sum_{f=u, d, s} \sigma_{f \bar{f}}$ rises from $r=0.25$ for $\mathbf{k}_{0}^{2}=2 \mathrm{GeV}^{2}$ to $r=0.53$ for $\mathbf{k}_{0}^{2}=8 \mathrm{GeV}^{2}$. This is easily understood since in the limit $m_{c}^{2} / \mathbf{k}_{0}^{2} \rightarrow 0$ the ratio $r$ should approach $2 / 3$ which follows from counting the electromagnetic charges. It is also noticeable that the decrease of the transverse cross section with increasing $\mathbf{k}_{0}^{2}$ is weaker in the case of charm production compared to the massless case. Stated differently, the $\mathbf{k}^{2}$-spectrum of the transverse contribution is flatter in the massive case. As a consequence the ratio of the longitudinal to the transverse contribution is decreasing with increasing $\mathbf{k}_{0}^{2}$ for charm production whereas for the massless flavors it is approximately constant. 


\begin{tabular}{|c|c|c|c|}
\hline & $\mathbf{k}_{0}^{2}=2 \mathrm{GeV}^{2}$ & $\mathbf{k}_{0}^{2}=4 \mathrm{GeV}^{2}$ & $\mathbf{k}_{0}^{2}=8 \mathrm{GeV}^{2}$ \\
\hline \hline \multicolumn{4}{|c|}{ DLA + corrections } \\
\hline \hline$\sigma_{T}^{e P}$ & 27 & 11 & 3.4 \\
\hline$\sigma_{L}^{e P}$ & 2.3 & 0.4 & 0.1 \\
\hline \hline$\sum_{i=T, L} \sigma_{i}^{e P}$ & 29.3 & 11.4 & 3.5 \\
\hline
\end{tabular}

Table 1: Results for total $e P$-cross sections (in pbarn) of diffractive dijet production for charm quarks.

\begin{tabular}{|c|c|c|c|}
\hline & $\mathbf{k}_{0}^{2}=2 \mathrm{GeV}^{2}$ & $\mathbf{k}_{0}^{2}=4 \mathrm{GeV}^{2}$ & $\mathbf{k}_{0}^{2}=8 \mathrm{GeV}^{2}$ \\
\hline \hline \multicolumn{4}{|c|}{ DLA + corrections } \\
\hline \hline$\sigma_{T}^{e P}$ & 108 & 30 & 6 \\
\hline$\sigma_{L}^{e P}$ & 9 & 2 & 0.6 \\
\hline \hline$\sum_{i=T, L} \sigma_{i}^{e P}$ & 117 & 32 & 6.6 \\
\hline
\end{tabular}

Table 2: Results for total $e P$-cross sections (in pbarn) of diffractive dijet production for three massless flavors.

The absolute numbers have been compared to the ones computed in a model based on nonperturbative twogluon exchange (soft pomeron model) [4] already in [16]. The soft pomeron model predicts cross sections which are reduced by a factor of approximately $1 / 2$ compared to our calculation. This difference is mainly due to the rise of the cross section at small $x_{\mathbb{P}}$ in our calculation according to the rise of the function $\left|x_{\mathbb{P}} G\left(x_{\mathbb{P}}, \Delta^{2}\right)\right|^{2}$. The soft pomeron model does not contain such a pronounced rise at small $x_{\mathbb{P}}$.

So far we have discussed jet cross sections. Since the large charm quark mass $m_{c}$ provides a hard scale $\Delta^{2}$ independent of the value of the transverse momentum $\mathbf{k}^{2}$ we can also extend the perturbative calculation to low values of $\mathbf{k}^{2}$. We can even integrate over the transverse momentum which gives us the possibility to calculate the charm contribution to the diffractive structure function $F_{2}^{D(3)}$ in our model. The latter has been introduced to describe diffractive events (events with a rapidity gap) in DIS. In terms of $F_{2}^{D(3)}$ the diffractive electron proton cross section reads

$$
\frac{d \sigma_{\mathrm{DIFF}}^{e P}}{d \beta d Q^{2} d x_{\mathbb{P}}}=\frac{2 \pi \alpha_{\mathrm{em}}^{2}}{\beta Q^{4}}\left[1+(1-y)^{2}\right] F_{2}^{D(3)}\left(\beta, Q^{2}, x_{\mathbb{P}}\right)
$$

where the longitudinal contribution has been neglected. With this definition of $d \sigma_{\text {DIFF }}^{e P}, F_{2}^{D(3)}$ can be obtained from the photon-proton cross section in the following way

$$
F_{2}^{D(3)}\left(\beta, Q^{2}, x_{\mathbb{P}}\right)=\frac{Q^{2}}{4 \pi^{2} \alpha_{e m}} \int_{0}^{\infty} d t \int_{0}^{M^{2} / 4-m_{c}^{2}} d \mathbf{k}^{2}\left[\frac{d \sigma_{T_{1}}^{\gamma^{*} P}}{d x_{\mathbb{P}} d \mathbf{k}^{2} d t}+\frac{d \sigma_{L}^{\gamma^{*} P}}{d x_{\mathbb{P}} d \mathbf{k}^{2} d t}\right] .
$$

where the cross sections in brackets are obtained from multiplying the expression for $t=0$ in eqs. (9) and (10) with the proton form factor $F_{P}\left(x_{\mathbb{P}}, t\right)$ and the factor $2 \pi$ (integration of $\phi$ ). In fig. 目 we display $F_{2}^{D(3)}$ as a function of $x_{\mathbb{P}}$. First of all, these curves demonstrate the rapid increase of the diffractive structure function at low values of $x_{\mathbb{P}}$. The fact that the shapes of the curves are not identical for different $\beta$ indicates the (weak) breaking of Regge factorization. Variation of $\beta$ leads to a variation of the $x_{\mathbb{P}}$-dependence. More precisely, a lowering of $\beta$ leads to an increase of the slope of the curve in accordance with the fact that the relevant scale of the gluon density is proportional to $1 /(1-\beta)$. For smaller $Q^{2}$ (left hand side) this effect is very small. By comparing the results for $Q^{2}=20 \mathrm{GeV}^{2}$ and $Q^{2}=50 \mathrm{GeV}^{2}$ one observes that a variation of $Q^{2}$ does not lead to a significant change of the absolute normalization of $F_{2}^{D(3)}$. This is demonstrated explicitly in fig. 6 where the $Q^{2}$-dependence of $F_{2}^{D(3)}$, respectively its transverse and longitudinal part, is 

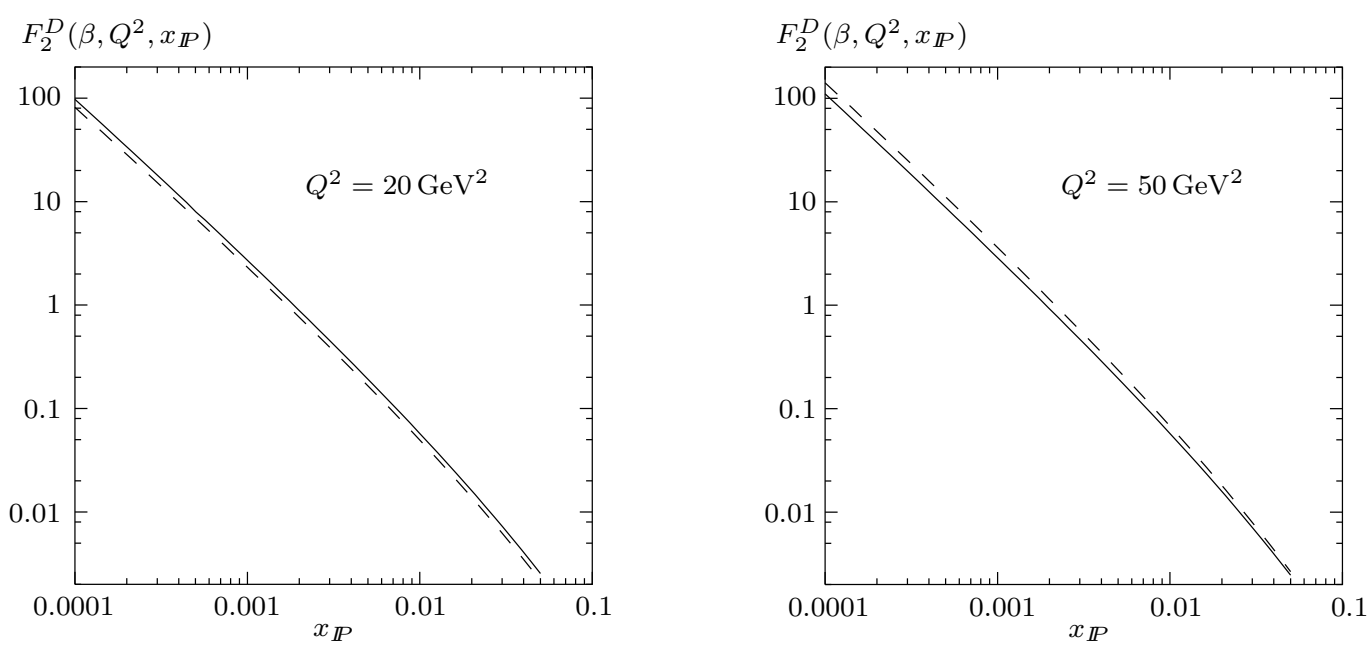

Figure 3: The $x_{\mathbb{P}}$-dependence of the diffractive structure function for $Q^{2}=20 \mathrm{GeV}^{2}, Q^{2}=50 \mathrm{GeV}^{2}$ and $\beta=1 / 3$, (solid line) and $\beta=2 / 3$ (dashed line).

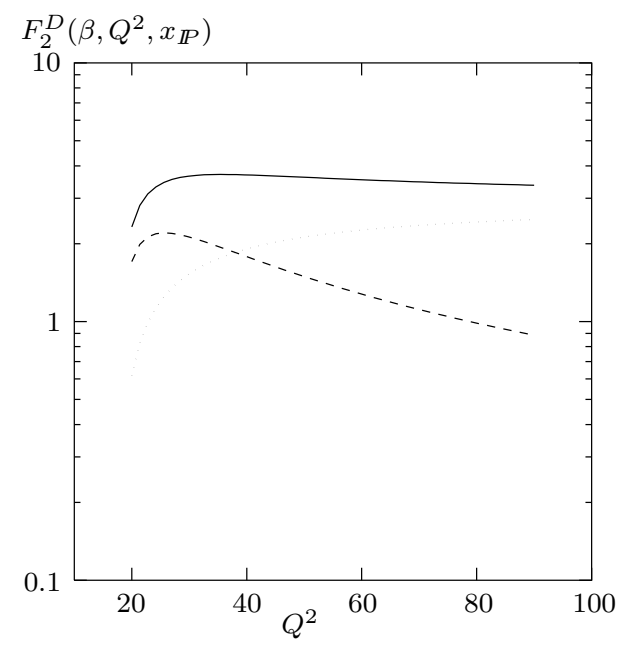

Figure 4: The $Q^{2}$-dependence of the diffractive structure function for $\beta=2 / 3$ and $x_{\mathbb{P}}=10^{-3}$. The dotted line is the transverse contribution the dashed line is the longitudinal part and the solid lines represent the sum.

displayed. One observes a weak logarithmic increase of the transverse contribution whereas the longitudinal part decreases rapidly with $Q^{2}$. The longitudinal part, in other words, is a higher twist contribution.

In fig. 司 we show $\beta$-spectra for two different values of $Q^{2}$. The most noticeable feature here is of course the charm threshold at the right end of each curve. One sees that near this threshold (for large $\beta$ ) the longitudinal contribution becomes comparable to or even larger than the transverse contribution. In the limit $\beta \rightarrow 0$ both the transverse and the longitudinal part tend to zero.

We can compare our results with experimental data on inclusive diffractive DIS to roughly estimate the relative contribution of the $c \bar{c}$-production. The ZEUS collaboration gives a value of $30-40$ for $F_{2}^{D(3)}\left(\beta, Q^{2}, x_{\mathbb{P}}\right)$ at $x_{\mathbb{P}}=10^{-3}, Q^{2}=16 \mathrm{GeV}^{2}$ and $\beta=0.65$ 17]. From the right hand side of fig. 5 we find a value of 

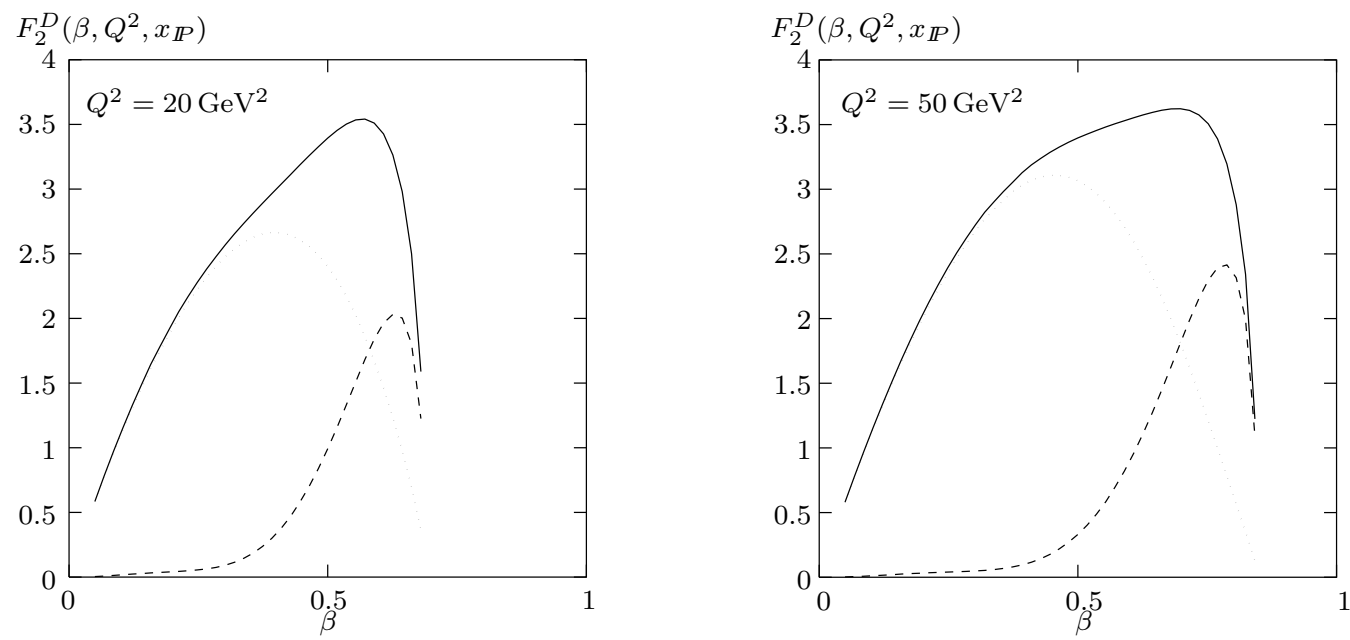

Figure 5: The $\beta$-dependence of the diffractive structure function for $Q^{2}=20 \mathrm{GeV}^{2}$ and $Q^{2}=50 \mathrm{GeV}^{2}$ and $x_{\mathbb{P}}=10^{-3}$. The dotted line is the transverse contribution the dashed line is the longitudinal part and the solid line represents the sum.

$F_{2}^{D(3)} \simeq 3.25$ for $x_{\mathbb{P}}=10^{-3}, Q^{2}=20 \mathrm{GeV}^{2}$ and $\beta \simeq 0.65$. We conclude that approximately $10 \%$ of the diffractive events observed at HERA in the corresponding kinematical region are due to $c \bar{c}$-production. A similar estimate has been given in [7. One should emphasize that the relative contribution of $c \bar{c}$ is a function of $x_{\mathbb{P}}$ due to the strong rise of the $c \bar{c}$-cross section at low $x_{\mathbb{P}}$. Since the cross section of the diffractive production of massless flavors is not expected to increase equally fast at low $x_{\mathbb{P}}$ due to the dominance of low momentum scales, the $c \bar{c}$ contribution is enhanced at small $x_{\mathbb{P}}$. The relative contribution of $10 \%$ seems to be large enough to expect the charm threshold to be observable in the experimenta data.

4.) To summarize, we have calculated the cross section for the diffractive production of an open $c \bar{c}$-pair in DIS. We have assumed two-gluon exchange and have treated both gluons perturbatively due to the large charm quark mass. In the double logarithmic approximation the cross section is proportional to the squared gluon density $\left|x_{\mathbb{P}} G\left(x_{\mathbb{P}}, \Delta^{2}\right)\right|^{2}$ of the proton with the scale $\Delta^{2}=\left(\mathbf{k}^{2}+m_{c}^{2}\right) /(1-\beta)$. We have first calculated jet cross sections where $\mathbf{k}^{2}$ is kept large and found that the charm contribution rises relative to massless flavours with increasing $\mathbf{k}^{2}$. For very high $\mathbf{k}^{2}$ the relative charm contribution follows from the electromagnetic charge factors. Since $m_{c}^{2}$ is sufficiently large the transverse momentum $\mathbf{k}^{2}$ can be integrated and the charm contribution to the diffractive structure function can be calculated in our model. As a function of $x_{\mathbb{P}}$ the diffractive structure function rises steeply at small $x_{\mathbb{P}}$ due to the rise of the gluon density. Since $\beta$ enters explicitly the scale of the latter our formulae display the breaking of Regge factorization. As a function of $Q^{2}$ the charm contribution to the diffractive structure function is approximately constant, i. e. it displays a leading-twist behavior. The most remarkable property of the $\beta$-spectrum is the charm threshold at large $\beta$. At small $x_{\mathbb{P}}$ where the charm contribution is relatively largest this threshold should be observable in the data. At small $\beta$ both the transverse and the longitudinbal cross section tend to zero.

As far as $c \bar{c}$-production is concerned our calculation is complete in the given approximation. The data on diffractive dissociation, however, show a constant behavior in the limit $\beta \rightarrow 0$. This shows that our model is not adequate to describe diffractive dissociation in the complete $\beta$-range. As an important correction the production of additional gluons has to be considered. Taking one additional gluon into account, one obtains a constant cross section at $\beta=0$. Corrections of this type have been investigated in [12, 18]. Their impact on diffractive charm production has been analyzed in [8]. The validity of the cited calculations has been limited to a restricted kinematical range. A complete calculation of the relevant corrections remains still an important task for future work. 
Acknowledgments: I have benefited very much from discussions with Prof. J. Bartels, Dr. M. Wüsthoff, Dr. M. Diehl and C. Ewerz. I am grateful to Prof. J. Bartels and C. Ewerz for carefully reading the manuscript. The support from the Studienstiftung des Deutschen Volkes is gratefully acknowledged.

\section{References}

[1] M.G.Ryskin, Z. Phys. C 57 (1993) 89;

S.Brodsky, L.Frankfurt, J.F.Gunion, A.H.Mueller, M.Strikman, Phys. Rev. D 50 (1994) 3134;

L.Frankfurt, W.Koepf, M.Strikman, Phys. Rev. D 54 (1996) 3194;

M.G.Ryskin, R.G.Roberts, A.D.Martin, E.M.Levin, hep-ph/9511228.

[2] J.Bartels, H.Lotter, M.Wüsthoff, Phys. Lett. B 379 (1996) 239;

Erratum, Phys. Lett. B 382 (1996) 449.

[3] J.Bartels, C.Ewerz, H.Lotter, M.Wüsthoff, Phys. Lett. B 386 (1996) 389.

[4] M.Diehl, Z. Phys. C 66 (1995) 181; preprint CPTH-S472-1096, hep-ph/9610430.

[5] N.N.Nikolaev, B.G.Zakharov, Phys. Lett. B 332 (1994) 177.

[6] M.G.Ryskin, M.Besancon, Proceedings of the HERA workshop 'Physics at HERA', Vol. 1 (edited by W.Buchmüller, G.Ingelman), Hamburg 1991.

[7] M.Genovese, N.N.Nikolaev, B.G.Zakharov, Phys. Lett. B 378 (1996) 347.

[8] E.M.Levin, A.D.Martin, M.G.Ryskin, T.Teubner, hep-ph/9606443.

[9] S.Catani, F.Hautmann, Nucl. Phys. B 427 (1994) 475;

S.Catani, M.Ciafaloni, F.Hautmann, Phys. Lett. B 242 (1990) 97; Nucl. Phys. B 366 (1991) 135.

[10] A.H.Mueller, Nucl. Phys. B 335 (1990) 115.

[11] N.N.Nikolaev, B.G.Zakharov, Z. Phys. C 53 (1992) 331.

[12] E.M.Levin, M.Wüsthoff, Phys. Rev. D 50 (1994) 4306.

[13] L.N.Lipatov, Sov. J. Nucl. Phys. 23 (1976) 642;

E.A.Kuraev, L.N.Lipatov, V.S.Fadin, Sov. Phys. JETP 44 (1976) 443; Sov. Phys. JETP 45 (1977) 199;

I.I.Balitskii, L.N.Lipatov, Sov. J. Nucl. Phys. 28 (1978) 822.

[14] A.Donnachie, P.V.Landshoff, Nucl. Phys. B 244 (1984) 322; Nucl. Phys. B 303 (1988) 634.

[15] M.Glück, E.Reya, A.Vogt, Z. Phys. C 67 (1995) 433.

[16] J.Bartels, M.Diehl, C.Ewerz, H.Lotter, M.Wüsthoff, in: Future Physics at HERA, Proceedings of the Workshop 1995/1996 (vol. 2), edited by G.Ingelman, A.DeRoeck, R.Klanner, Hamburg 1996.

[17] ZEUS collaboration, Z. Phys. C 70 (1996) 391.

[18] J.Bartels, M.Wüsthoff, J. Phys. G 22 (1996) 929. 